\title{
黄柳与垂柳的耐热性和耐旱性比较研究
}

\author{
杨甲定 赵哈林 张铜会 \\ (中国科学院寒区早区环境与工程研究所,兰州 730000)
}

\begin{abstract}
摘 要 作为一种优良的固沙植物, 黄柳 (Salix gordejevii) 主要分布于内蒙古科尔沈沙地和浑善达克沙地, 在流动沙 丘边缘和半流动沙丘生长最多, 属于灌木型先锋植物。实验对黄柳的耐热性和耐旱性与垂柳 (Salix babylonica) 进行 了比较研究。两种植物的离体叶片在 6 个温度 (即 25、30、35、40、45、50 ${ }^{\circ} \mathrm{C}$ ) 下处理 $1 \mathrm{~h}$; 在 6 个水势梯度 (即 $0 、$ $-0.25 、-0.5 、-0.75 、-1.0 、-1.25 \mathrm{MPa}$ )下处理 $24 \mathrm{~h}$ 后, 测定了其含水量、膜透性、光系统 II 的最大光化学效率 (由 $F_{\mathrm{v}} / F_{\mathrm{m}}$ 计算) 等参数。黄柳叶片在温度处理和干旱处理中均能保持比垂柳较高的含水量; $45{ }^{\circ} \mathrm{C}$ 以下的温度对两 种植物叶片的膜透性没有明显影响, 但高于 $45^{\circ} \mathrm{C}$ 的温度 (本实验中为 $50{ }^{\circ} \mathrm{C}$ ) 使黄柳和垂柳叶片膜透性剧增, 且黄柳 增大更多; 干旱处理造成两种植物叶片膜透性增大, 且垂柳的总是大于黄柳的; 低于 $35{ }^{\circ} \mathrm{C}$ 的温度对 $F_{\mathrm{v}} / F_{\mathrm{m}}$ 没有影 响, 高于 $40{ }^{\circ} \mathrm{C}$ 的温度使两种植物叶片的 $F_{\mathrm{v}} / F_{\mathrm{m}}$ 明显降低, 且黄柳的极显著地小于垂柳的; 干旱处理总使两种植物 叶片的 $F_{\mathrm{v}} / F_{\mathrm{m}}$ 降低, 当渗透势小于 $-0.75 \mathrm{MPa}$ 时, 黄柳的 $F_{\mathrm{v}} / F_{\mathrm{m}}$ 显著地大于垂柳的。这些结果说明黄柳的耐旱性 比垂柳强, 其耐热性比垂柳差。就光合器官而言, 黄柳、垂柳耐热性和耐旱性的差别主要是由于通过光系统 II 的电 子传递对高温和干旱的敏感性不同造成的。
\end{abstract}

关键词 温度处理 干旱处理 含水量 膜透性 叶绿素苂光 $F_{\mathrm{v}} / F_{\mathrm{m}}$

\section{A COMPARATIVE STUDY ON HEAT AND DROUGHT TOLERANCE BETWEEN SALIX GORDEJEVII AND SALIX BABYLONICA}

\author{
YANG Jia-Ding ZHAO Ha-Lin and ZHANG Tong-Hui \\ ( Cold \& Arid Regions Environmental and Engineering Research Institute, Chinese Academy of Sciences, Lanzhou 730000, China)
}

\begin{abstract}
Salix gordejevii (SG), a useful dune-fixing plant, is a pioneer bush that establishes primarily on the margins of moving sand dunes in Horqin Sand Land and Hunshandak Sand Land, Inner Mongolia, China. In this study, the heat and drought tolerance of SG were examined and compared to $S$. babylonica (SB). Leaves removed from the two species were subjected to six temperature treatments $(25,30,35,40,45,50$ ${ }^{\circ} \mathrm{C}$ ) for 60 minutes and six drought treatments $(0,-0.25,-0.5,-0.75,-1.0,-1.25 \mathrm{MPa})$ for 24 hours. The relative water content, membrane permeability and maximal quantum efficiency of photosystem II ( PSII) were assayed. Leaves of SG maintained more water during both temperature and drought treatments. Temperature treatments below $45{ }^{\circ} \mathrm{C}$ had no effect on the membrane permeability of either of the two species, but treatments above $45{ }^{\circ} \mathrm{C}$ induced much higher electrolyte leakage in SG than in SB. Each drought treatment caused an increase in membrane permeability in both SG and SB but was greater in SB in all drought treatments. Temperatures below $35{ }^{\circ} \mathrm{C}$ did not influence maximal quantum efficiency of PS II (measured as $F_{\mathrm{v}} /$ $F_{\mathrm{m}}$ ) in either of the two species, whereas treatments above $40{ }^{\circ} \mathrm{C}$ caused $F_{\mathrm{v}} / F_{\mathrm{m}}$ to decrease in both species but was significantly lower in SG. Drought treatments resulted in a decline in $F_{\mathrm{v}} / F_{\mathrm{m}}$ in both species but declines were significantly higher in SG when the osmotic potential was lower than $-0.75 \mathrm{MPa}$. These results suggest that SG has a higher capacity for drought tolerance and a lower capacity for heat tolerance compared to SB. The different stress tolerance between SG and SB was due mainly to different sensitivities of electron transport through PSII to heat and drought.
\end{abstract}

Key words Temperature treatment, Osmotic treatment, Relative water content, Membrane permeability, Chlorophyll fluorescence, Photochemical efficiency

生长在沙漠地区的植物经常面临午间高温和水 分亏缺的考验 (Hopkins, 1999), 它们对高温和干旱 及其它环境因子的耐受能力很大程度上决定其分布
甚至生存 (Lack \& Evans, 2002)。由于沙区高温和 水分亏缺经常相伴发生, 在野外条件下很难剥离开 来(Hopkins, 1999), 但耐热性和耐旱性毕竟是两种 
不同的抗逆性, 例如, 玉米 (Zea mays) 等的耐热性 强, 抗旱性不强; 向日葵( Helianthus annus) 等抗旱性 强, 却不耐高温 (潘瑞炽和董愚得, 1995), 因此有必 要在室内条件下对某些沙区植物的耐热性和耐旱性 进行比较研究。

黄柳 (Salix gordejevii) 与垂柳 ( S. babylonica) 同 属杨柳科柳属。黄柳主要分布于科尔沁沙地和浑善 达克沙地, 是该地区经济价值较高的固沙植物 (常学 礼等, 1993), 在流动沙丘边缘和半流动沙丘分布最 多, 属于灌木型先锋植物 (刘新民等, 1996), 已引种 到毛乌素沙地、腾格里沙漠, 在无灌溉的条件下仍生 长良好。常学礼等(1993)对黄柳的生长进程及地上 生物量动态进行了研究; 周海燕和赵爱芬 (1999)测 定了黄柳叶片的气孔变化特征, 发现下表皮气孔受 环境因子和季节变化的影响较大, 并认为黄柳的耐 旱性与季节、沙丘位置有关系。但对黄柳适应沙区 环境的生理机制尚无深入系统的研究。垂柳在中国 北方大部分地区均有分布, 作为一种普通树种, 在科 尔沁沙地居民区也有一些种植。本研究即以中国科 学院奈曼沙漠化试验研究站附近的黄柳和垂柳为实 验材料, 研究比较了二者对当地高温、干旱两个主要 生态环境因子的耐受能力, 以期能从生理学角度对 两种植物的引种或种植条件提出一点有益的建议。

\section{1 材料和方法}

\section{1 样地与材料处理}

中国科学院奈曼沙漠化试验研究站位于内蒙古 自治区东部科尔沁沙地的奈曼旗境内, 地理位置为 $120^{\circ} 42^{\prime} \mathrm{E}, 42^{\circ} 55^{\prime} \mathrm{N}$, 海拔 $358 \mathrm{~m}$ 左右。植物生长季 ( 5 $\sim 8$ 月) 的主要环境特点为干旱、午间高温和强光 照。

于 2003 年 8 月中旬分别在研究站植物引种区 内和附近居民区选定灌溉充分、生长良好的黄柳灌 从和垂柳植株。选择晴天上午 6:00, 从所选黄柳、 垂柳上剪取发育良好的当年生成熟枝条, 切端插于 蒸馏水中, 迅速带回实验室。在室内蒸馏水中浸泡 $4 \mathrm{~h}$ 后, 进行人工模拟的温度、干旱处理。

参照 Law 和 Crafts-Brandner（1999）报道的快速 热胁迫处理方法, 每种植物选择形态一致的成熟叶 片, 用浸湿的脱脂棉包裹其叶柄, 分成 6 组 (每组 3 片叶子), 分别置于铺有湿润滤纸的 6 个培养皿中, 然后放入已预设温度的植物组织培养箱, 暗中处理 $60 \mathrm{~min}$ 。6 组温度设置分别为: $25 、 30 、 35 、 40 、 45 、 50$ ${ }^{\circ} \mathrm{C}$, 进行 3 次重复。
参照王娟和李德全 (2002) 的方法, 以蒸馏水作 为 $0 \mathrm{MPa}$ 的对照, 配制渗透势分别相当于 -0.25 $\mathrm{MPa} 、-0.5 \mathrm{MPa} 、-0.75 \mathrm{MPa} 、-1.0 \mathrm{MPa}$ - 1.25 MPa 的 PEG6000 溶液。将每种植物叶片剪取 6 组 (每组 3 片), 叶柄插于水或 PEG6000 溶液中, 在设置 为 $25{ }^{\circ} \mathrm{C}$ 的植物组织培养箱内暗中放置 $24 \mathrm{~h}$, 进行干 旱处理, 3 次重复。

\section{2 生理参数的测定}

温度和干旱处理前, 用十万分之一电子分析天 平 (Analytical Plus Electronic Balance AP250D, Ohaus 公司, USA) 准确测定每组叶片的质量 $\mathrm{M}_{0}$ 。处理结束 后, 在暗室中用便携式荧光仪 (Handy PEA, Hansate$\mathrm{ch}$ 公司, UK) 对叶片进行荧光测定。 $F_{0}$ (原初荧光 值)、 $F_{\mathrm{m}}$ (经 $0.8 \mathrm{~s} 3000 \mu \mathrm{mol} \cdot \mathrm{m}^{-2} \cdot \mathrm{s}^{-1}$ 饱和光脉冲激 发后的最大荧光值) 由仪器自动记录。光系统 II 的 最大光化学效率 $\left(F_{\mathrm{v}} / F_{\mathrm{m}}\right)$ 由 $\left(F_{\mathrm{m}}-F_{0}\right) / F_{\mathrm{m}}$ 计算。 随即测定叶片质量 $M$, 计算温度或干旱处理后叶片 的相对含水量 $R W C=M / M_{0} \times 100 \%$ 。然后参照 Meyer 和 Santarius（1998）的方法, 将叶片完整浸泡 于 $30 \mathrm{ml}$ 蒸馏水中, 在 $25{ }^{\circ} \mathrm{C}$ 植物组织培养箱内暗中 振荡 $12 \mathrm{~h}$, 用 P4 多功能测定仪 (Multiline P4 Universal Meter, WTW 公司, Germany) 测定其电导率 ( $\mu$ s $\mathrm{cm}^{-1}$ ), 结合处理后叶片的质量 $M$, 通过电解质的渗 漏 (Electrolyte leakage, EL) 比较叶片组织经温度或干 旱处理后的细胞膜透性 $\left(\mu \mathrm{s} \cdot \mathrm{cm}^{-1} \cdot \mathrm{g}^{-1}\right)$ 。

\section{3 统计分析}

实验数值由图显示。如果标准差小于其对应平 均值的 $5 \%$, 则不在图中显示出来。同一处理下, 黄 柳和垂柳某一参数之间的差异显著性用 $t$-检验 (EXCEL 统计程序) 完成。分析前, 所比较的数据组 先检验其方差齐性 ( $F$-检验)。

\section{2 实验结果}

\section{1 温度和干旱处理对叶片含水量的影响}

从图 1a 可以看出, 两种植物的叶片在温度处理 过程中, 其相对含水量随温度的升高均有微弱的降 低, 即黄柳从 $100 \%\left(25{ }^{\circ} \mathrm{C}\right)$ 降到 $96.10 \%\left(50{ }^{\circ} \mathrm{C}\right)$, 垂 柳从 $100 \%\left(25{ }^{\circ} \mathrm{C}\right)$ 降到 $95.86 \%\left(50{ }^{\circ} \mathrm{C}\right)$, 在各个温 度处理下, 黄柳叶片的相对含水量均大于垂柳的。 干旱处理导致两种植物叶片的相对含水量明显下降 (图 1b), 而且黄柳叶片相对含水量总是大于垂柳叶 片。以上结果说明不论在温度处理还是干旱处理 下, 黄柳叶片均具有较高的保持水分的能力, 由此可 以初步认定黄柳比垂柳具有较高的抗旱能力。 
2.2 温度和干旱处理对叶片细胞膜透性的影响

$45{ }^{\circ} \mathrm{C}$ 以下的温度对两种植物叶片的膜透性没 有明显影响(图 $2 \mathrm{a}$ ), 基本保持在 $25 \mu \mathrm{s} \bullet \mathrm{cm}^{-1} \cdot \mathrm{g}^{-1}$ 左 右, 但黄柳膜透性略高于垂柳。经 $50{ }^{\circ} \mathrm{C}$ 处理后, 黄 柳的膜透性增加约 10 倍, 为 $262.32 \mu \mathrm{s} \cdot \mathrm{cm}^{-1} \cdot \mathrm{g}^{-1}$, 垂柳则增加约 3 倍, 为 $75.77 \mu \mathrm{s} \cdot \mathrm{cm}^{-1} \cdot \mathrm{g}^{-1}$, 说明 $50{ }^{\circ} \mathrm{C}$ 处理对黄柳叶片的细胞膜造成了较大的伤害, 亦即黄柳叶片的细胞膜较垂柳不耐高温。由图 $2 \mathrm{~b}$ 可见, 两种植物叶片的膜透性对干旱的响应趋势很 相似, 随胁迫强度的增加而增加 $(-1.25 \mathrm{MPa}$ 处理 例外), 但垂柳的膜透性总是大于黄柳, 说明在干 旱处理中垂柳遭受到更严重的伤害，亦即垂柳的耐

\section{旱性较黄柳差。}

2.3 温度和干旱处理对叶片最大光化学效率的影 响

从图 3a 可以看出, $35{ }^{\circ} \mathrm{C}$ 以下的温度处理, 黄柳 和垂柳叶片的最大光化学效率没有差别, 而 $40{ }^{\circ} \mathrm{C}$ 以上的温度处理后, 垂柳的最大光化学效率极显著 地大于黄柳叶片。图 $3 b$ 表明, 渗透势 $\geqslant-0.5 \mathrm{MPa}$ 的干旱处理，尽管黄柳与垂柳叶片的最大光化学效 率均轻微下降，但无明显差别; 渗透势 $\leqslant-0.75$ $\mathrm{MPa}$ 的干旱处理，导致垂柳叶片的最大光化学效率 明显降低，且与黄柳有显著差异。
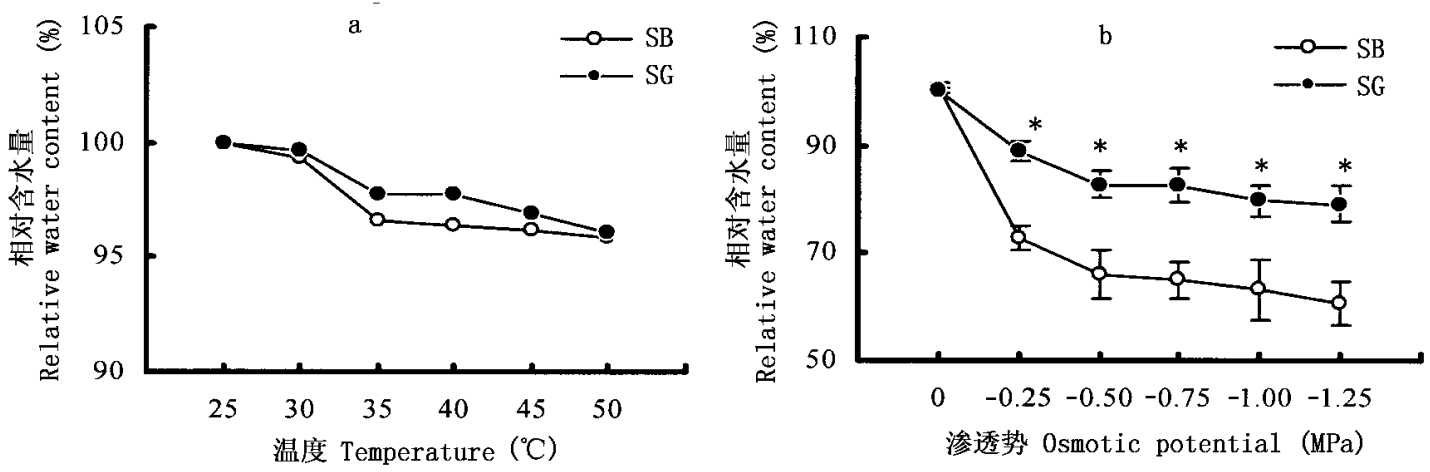

图 1 温度处理 (a)和干旱处理 (b) 对黄柳和垂柳叶片相对含水量的影响

Fig. 1 Relative water content of leaves of Salix gordejevii and $S$. babylonica after temperature treatment for $1 \mathrm{~h} \mathrm{(a)} \mathrm{and} \mathrm{drought} \mathrm{treatment} \mathrm{for} 24 \mathrm{~h}(\mathrm{~b})$ SG: 黄柳 Salix gordejevii SB: 垂柳 $S$. babylonica 图 b 中坚线为标准差 $S D(n=3) \mathrm{In} \mathrm{b}$, vertical bars indicate the standard deviation $(n=3)$ $*: p<0.05 * *: p<0.01$
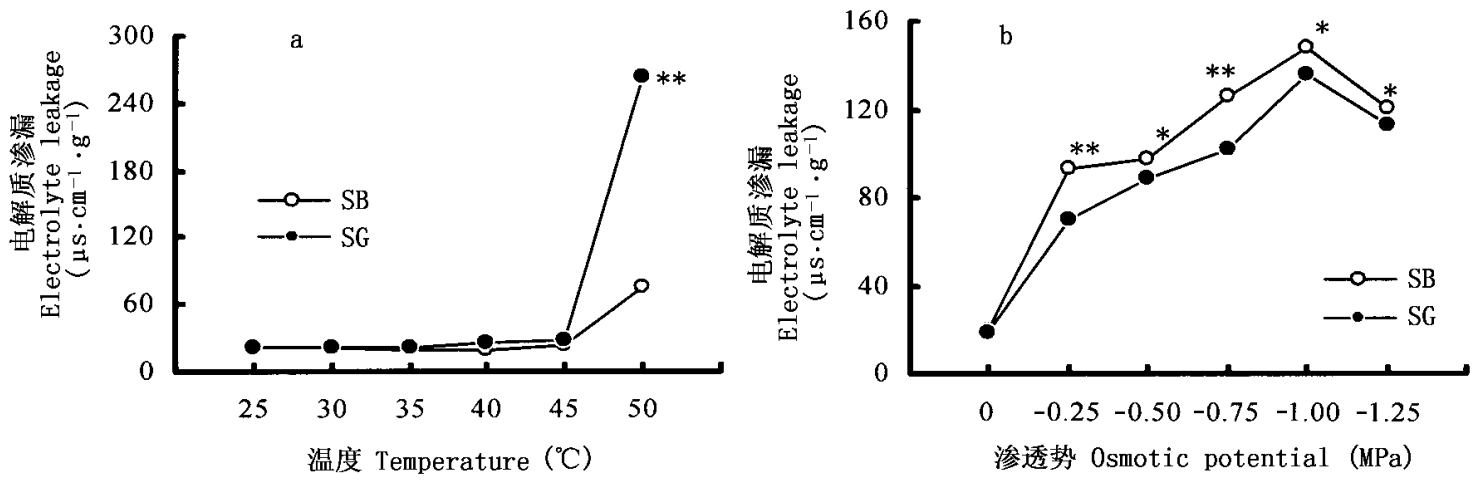

图 2 温度和干旱处理后黄柳和垂柳叶片的膜透性变化

Fig.2 Cell membrane permeability in leaves of Salix gordejevii and $S$. babylonica after temperature treatment for $1 \mathrm{~h}$ (a) and drought treatment for $24 \mathrm{~h}$ (b) $\mathrm{SG}, \mathrm{SB}, *, * *$ : 同图 1 See Fig. 1 

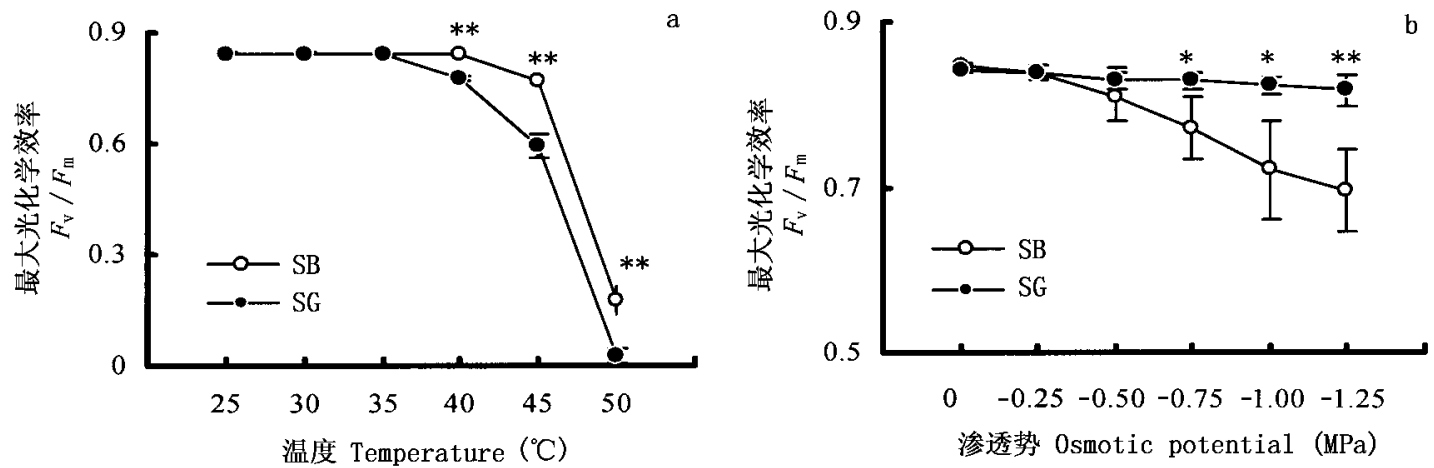

图 3 温度和干旱处理后黄柳和垂柳叶片的最大光化学效率

Fig.3 The maximal quantum yield of PS II $\left(F_{\mathrm{v}} / F_{\mathrm{m}}\right)$ in leaves of Salix gordejevii and $S$. babylonica after temperature treatment for $1 \mathrm{~h} \mathrm{(a)} \mathrm{and} \mathrm{drought} \mathrm{treatment} \mathrm{for} 24 \mathrm{~h}(\mathrm{~b})$

$\mathrm{SG}, \mathrm{SB}, *, * *$ : 同图 1 See Fig.1 图 b 中坚线为标准差 $S D(n=9)$ In b, vertical bars indicate the standard deviations $(n=9)$

2.4 温度和干旱处理对叶片原初荧光 $\left(F_{0}\right)$ 和最大 菼光 $\left(F_{\mathrm{m}}\right)$ 的影响

无论黄柳或垂柳, 低于 $45{ }^{\circ} \mathrm{C}$ 的温度处理没有导 致叶片 $F_{0}$ 的明显变化, $50{ }^{\circ} \mathrm{C}$ 的温度处理则造成 $F_{0}$ 从(635 \pm 4 ) 剧增至 $(865 \pm 2$ ) (图 4a); 尽管黄柳和垂
柳叶片的 $F_{\mathrm{m}}$ 均是温度较低时保持稳定, 高温时明 显下降, 但实验中出现显著下降时的温度并不相同, 垂柳为大于 $40{ }^{\circ} \mathrm{C}$, 黄柳为大于 $35{ }^{\circ} \mathrm{C}$ 。

图 4c 表明, 经干旱处理的黄柳和垂柳叶片, 其 $F_{0}$ 在各个水势梯度中基本保持稳定, 黄柳为
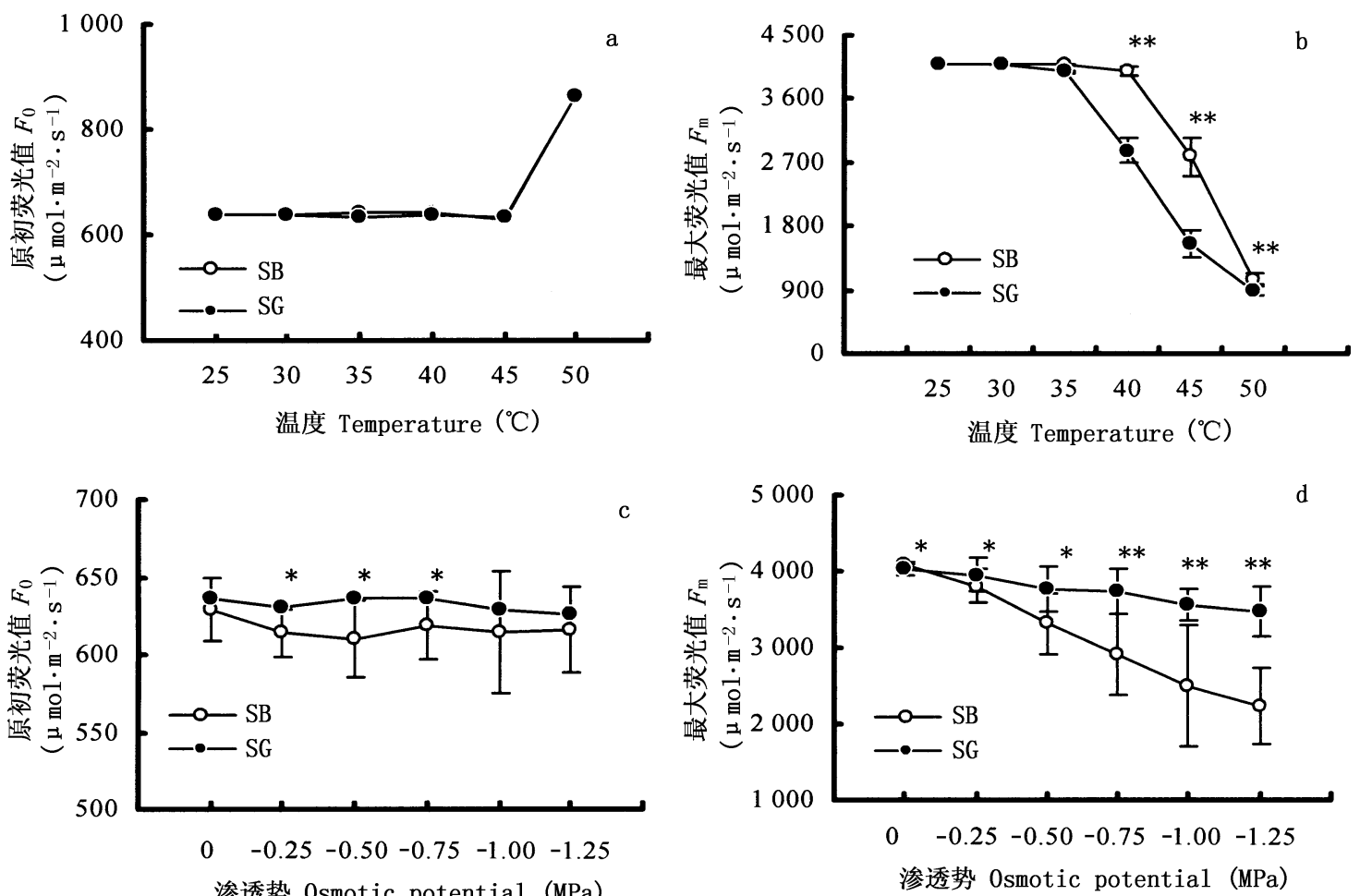

渗透势 0smotic potential (MPa)

图 4 温度和干旱处理后黄柳和垂柳叶片的原初苂光 $\left(F_{0}\right)$ 和最大苂光 $\left(F_{\mathrm{m}}\right)$

Fig.4 The values of the initial fluorescence $\left(F_{0}\right)$ and the maximal fluorescence $\left(F_{\mathrm{m}}\right)$ in leaves of Salix gordejevii and $S$. babylonica after temperature treatment for $1 \mathrm{~h}(\mathrm{a}, \mathrm{b})$ and drought treatment for $24 \mathrm{~h}(\mathrm{c}, \mathrm{d})$

$\mathrm{SG}, \mathrm{SB}, *, * *$ : 同图 1 See Fig. 1 图中坚线为标准差 $S D(n=9)$ 。图 a 中, $\mathrm{SG}$ 和 $\mathrm{SB} 50{ }^{\circ} \mathrm{C}$ 处理中的标准偏差分别为 \pm 67 和 \pm 112 , 其它 温度处理的标准偏差均小于各自平均值的 5\%。图 $\mathrm{c}$ 中, SG 的标准差都小于其平均值的 5\% Vertical bars indicate the standard deviations $(n=9)$. In a, the standard deviation in $50{ }^{\circ} \mathrm{C}$ treatments were \pm 67 and \pm 112 for SG and SB respectively, standard deviations in other temperature treatments were less than $5 \%$ of their means. In c, all standard deviations in SG were less than $5 \%$ of their means 
(632 \pm 4$)$, 垂柳为 $(617 \pm 7)$; 无论黄柳或垂柳, 其 $F_{\mathrm{m}}$ 均随处理水势的增加逐渐降低, 但垂柳叶片的降低 幅度明显大于黄柳, 当渗透势 $\leqslant-0.75 \mathrm{MPa}$ 的干旱 处理中, 垂柳叶片的 $F_{\mathrm{m}}$ 极显著地小于黄柳叶片。

\section{3 讨 论}

植物细胞膜是许多环境胁迫因子的作用靶位之 一, 普遍认为, 能在胁迫条件下保持细胞膜的完整性 和稳定性是植物抗逆性的一个重要成分 (Bajji et $a l$, 2002)。而细胞膜的伤害程度很容易通过检测 电解质的外渗来估算。这一方法已用于计算多种非 生物胁迫对细胞膜造成的伤害, 如干旱 (Bajji et $a l ., 2002$ )、低温( Tamura, 2000)、高温( Ismail \& Hall, 1999; Liu \& Huang, 2000)、盐胁迫 (Chen et al. , 1999) 等。在本实验中, $45{ }^{\circ} \mathrm{C}$ 以下的温度对两种植 物叶片的膜透性没有明显影响, 经 $50{ }^{\circ} \mathrm{C}$ 处理后, 黄 柳叶片的膜透性远高于垂柳 (图 2a), 说明黄柳的耐 热性较垂柳差。由图 $2 b$ 可见, 两种植物叶片的膜 透性对干旱的响应趋势很相似, 但垂柳的膜透性总 是大于黄柳, 说明在干旱处理中垂柳叶片的细胞膜 遭受了更严重的伤害, 亦即垂柳的耐旱性较黄柳 差。

叶绿素苂光检测可以灵敏快速地了解植物光合 作用对外界环境因子的响应 (Maxwell \& Johnson, 2000), 如干旱 (Ögren, 1990; Epron et al., 1992; Lu \& Zhang, 1998; 1999)、高温 (Liu \& Huang, 2000; Lu \& Zhang, 2000; Crafts-Brandner \& Law, 2000; CraftsBrandner \& Salvucci, 2002)、低温（DeEll \& Toivonen, 1999)、强光 (Hamerlynck, 2001)、紫外辐射 (Jansen et $a l ., 2001$ ) 等。植物叶片的最大光化学效率 $F_{\mathrm{v}} / F_{\mathrm{m}}$, 作为光系统 II 潜在光化学活性的度量, 非胁 迫条件下基本在 0.83 左右, 不受物种的影响, 对环 境变化非常敏感, 在胁迫条件下该参数明显下降, 表 明有功能的反应中心含量降低（Krause \& Weis, 1991)。从图 3a 和 $\mathrm{b}$ 可见, $40{ }^{\circ} \mathrm{C}$ 以上的温度处理后, 垂柳的最大光化学效率极显著地大于黄柳叶片, 说 明垂柳叶片光合器官的耐高温能力高于黄柳; 而渗 透势 $\leqslant-0.75 \mathrm{MPa}$ 的干旱处理, 导致垂柳叶片的最 大光化学效率明显降低, 且显著小于黄柳叶片的, 说 明黄柳叶片的光合器官具有较强的抗干旱胁迫的能 力, 这与膜透性分析得出的结果相一致。同时, 比 较图 3a 和 $\mathrm{b}$ 可以发现, $1 \mathrm{~h}$ 的高温处理对植物叶片 最大光化学效率的影响要远大于 $24 \mathrm{~h}$ 的干旱处理, 例如, 垂柳叶片经 $50{ }^{\circ} \mathrm{C}$ 处理后, $F_{\mathrm{v}} / F_{\mathrm{m}}$ 是 $25{ }^{\circ} \mathrm{C}$ 处理
组的 $20.8 \%$, 而经渗透势为 $-1.25 \mathrm{MPa}$ 的干旱处理 后, 垂柳的 $F_{\mathrm{v}} / F_{\mathrm{m}}$ 是 $0 \mathrm{MPa}$ 对照组的 $82.2 \%$, 说明 高温对植物组织的影响要比干旱迅速而且严重。

叶绿素原初苂光 $\left(F_{0}\right)$ 的增高被认为是光系统 II反应中心的破坏或可逆失活造成的（Krause \& Weis, 1991; Demmig et al., 1987), 最大苂光 $\left(F_{\mathrm{m}}\right)$ 可 以反映通过光系统 II 的电子传递情况 (Lichtenthaler, 1988; 张守仁, 1999)。从图 4a 和 $\mathrm{b}$ 可知, 小 于 $45{ }^{\circ} \mathrm{C}$ 的处理中, 两种植物叶片的原初荧光没有明 显变化, 最大光化学效率的降低主要是由于 $F_{\mathrm{m}}$ 的 降低, 即通过光系统 II 的电子传递受到阻抑; 大于 $45{ }^{\circ} \mathrm{C}$ 的处理导致两种植物叶片最大光化学效率的 降低, 一方面是与通过光系统 II 的电子传递受阻, 也 与光系统 II 反应中心的失活或破坏有关。同时说 明, 黄柳叶片的耐热性低于垂柳的, 主要是其电子传 递的耐热性较低所致, 二者的光系统 II 反应中心的 耐热性在本实验条件下没有明显差别 (图 4a)。各 干旱处理下两种植物叶片的 $F_{0}$ 没有明显增高, 最 大光化学效率的降低完全是由于 $F_{m}$ 的降低, 即通 过光系统 II 的电子传递受阻造成的。综合温度处理 和干旱处理的叶绿素苂光变化, 可知黄柳、垂柳叶片 中通过光系统 II 的电子传递对高温和干旱的敏感性 不同, 从而造成了两种植物其叶片光化学活性对高 温和干旱的耐受能力产生差异。

综上所述, 虽然同属杨柳科柳属, 但黄柳的耐旱 性强于垂柳, 其耐热性低于垂柳。因此, 黄柳作为固 沙植物引种时, 还需要考虑引种地的日间温度, 如果 该地温度过高 (比如热带沙漠地区), 很可能导致引 种失败。而垂柳由于不耐干旱, 对水分供应要求较 高, 不适宜在沙漠缺水地区种植。

\section{参 考 文 献}

Bajji M, Kinet JM, Lutts S (2002) . The use of the electrolyte leakage method for assessing cell membrane stability as a water stress tolerance test in durum wheat. Plant Growth Regulation, 36, 61 -70 .

Chang XL(常学礼), Li SG (李胜功), Zhao XY (赵学勇), Zhang WJ (张维静) (1993). Study on the growth and aboveground biomass characteristics of Salix gordejivii. In: Liu XM (刘新民), Zhao HL(赵哈林) eds. Study on Comprehensive Renovation of Ecological Environment in Horqin Sandy Land (科 尔泌沙地生态环境综合整治研究). Gansu Science and Technology Press, Lanzhou, 201 - 208. (in Chinese)

Chen Q, Zhang WH, Liu YL (1999). Effect of NaCl, glutathione and ascorbic acid on function of tonoplast vesicles isolated from barley leaves. Journal of Plant Physiology, 155, 685-690.

Crafts-Brandner SJ, Salvucci ME (2002). Sensitivity of photosynthesis in a $\mathrm{C}_{4}$ plant, maize, to heat stress. Plant Physiology, 129, 1773 - 1780 . 
Crafts-Brandner SJ, Law RD (2000). Effect of heat stress on the inhibition and recovery of the ribulose-1,5-bisphosphate carboxylase/oxygenase activation state. Planta, 212, $67-74$.

DeEll JR, Toivonen PMA (1999). Chlorophyll fluorescence as an indicator of physiological changes in cold-stored broccoli after transfer to room temperature. Journal of Food Science, 64, 501 -503 .

Demmig B, Winter K, Kruger A, Czygan FC (1987) . Photoinhibition and zeaxanthin formation in intact leaves. A possible role of the xanthophylls cycle in the dissipation of excess light energy. Plant Physiology, 84, 218 - 224.

Epron D, Dreyer E, Bréda N (1992). Photosynthesis of oak trees (Quercus petraea (Matt) Liebl.) during drought stress under field conditions: diurnal course of net $\mathrm{CO}_{2}$ assimilation and photochemical efficiency of photosystem II . Plant, Cell and Environment, $15,809-820$.

Hamerlynck EP (2001). Chlorophyll fluorescence and photosynthetic gas exchange responses to irradiance of tree of heaven (Ailanthus altissima) in contrasting urban environments. Photosynthetica, 39: $79-86$.

Hopkins WG (1999). Introduction to Plant Physiology 2nd edn. John wiley \& Sons Inc., New York, 463-464.

Ismail AM, Hall AE (1999). Reproductive-stage heat tolerance, leaf membrane thermostability and plant morphology in cowpea. Crop Science, 39, $1762-1768$.

Jansen MAK, Van den Noort RE, Tan MYA, Prinsen E, Lagrimini LM, Thorneley RNF (2001). Phenol-oxidizing peroxidases contribute to the protection of plants from ultraviolet radiation stress. Plant Physiology, 126, 1012 - 1023.

Krause GH, Weis E (1991). Chlorophyll fluorescence and photosynthesis: the basics. Annual Review of Plant Physiology and Plant Molecular Biology, 42, 313 - 349.

Lack AJ, Evans DE (2002). Instant Notes in Plant Biology. Science Press, Beijing, 161 .

Law RD, Crafts-Brandner SJ (1999). Inhibition and acclimation of photosynthesis to heat stress is closely correlated with activation of ribulose-1, 5-bisphosphoate carboxylase/oxygenase. Plant Physiology, 120, $173-181$.

Lichtenthaler HK (1988). Application of Chlorophyll Fluorescence in Photosynthetic Research, Stress Physiology, Hydrobiology and Remote Sensing. Kluwer Academic, Dordrecht, 129 - 142 .

Liu XM (刘新民)，Zhao HL (赵哈林)，Zhao AF (赵爱芬)
(1996). Wind-Sandy Environment and Vegetation in the Horqin Sandy Land, China (科尔泌沙地风沙环境与植被). Science Press, Beijing, 77 - 78. (in Chinese)

Liu XZ, Huang BR (2000). Heat stress injury in relation to membrane lipid peroxidation in creeping bentgrass. Crop Science, 40, $503-510$.

Lu CM, Zhang JH (1998). Effects of water stress on photosynthesis, chlorophyll fluorescence and photoinhibition in wheat plants. Australian Journal of Plant Physiology, 25, 883-892.

Lu CM, Zhang JH (1999) . Effects of water stress on photosystem II photochemistry and its thermostability in wheat plants. Journal of Experimental Botany, 50, 1199 - 1206.

Lu CM, Zhang JH (2000) . Heat-induced multiple effects on PSII in wheat plants. Journal of Plant Physiology, 156, $259-265$.

Maxwell K, Johnson GN (2000). Chlorophyll fluorescence - a practical guide. Journal of Experimental Botany, 51, 659-668.

Meyer H, Santarius KA (1998). Short-term thermal acclimation and heat tolerance of gametophytes of mosses. Oecologia, 115, 1 -8 .

Ögren E (1990) . Evaluation of chlorophyll fluorescence as a probe for drought stress in willow leaves. Plant Physiology, 93, 1280 -1285 .

Pan RC(潘瑞炽), Dong YD(董愚得) (1995). Plant Physiology (植物生理学)3rd edn. Higher Education Press, Beijing, 329. (in Chinese)

Tamura A (2000). Evaluation of freezing tolerance of whole plants in komatsuna (Brassica campestris L.) and spinach (Spinacia oleraceae L. ). Journal of Japanese Society of Horticulture Science, $69,332-338$.

Wang J(王娟)，Li DQ(李德全)（2002）Effects of water stress on AsA-GSH cycle and $\mathrm{H}_{2} \mathrm{O}_{2}$ content in maize root. Chinese Journal of Eco-Agriculture（中国生态农业学报), 10 (2), 94-96. (in Chinese with English abstract)

Zhang SR(张守仁) (1999). A discussion on chlorophyll fluorescence kinetics parameters and their significance. Chinese Bulletin of Botany (植物学通报), 16, 444 - 448. (in Chinese with English abstract)

Zhou HY(周海燕), Zhao AF(赵爱芬) (1999). Seasonal dynamics of stomatal regulation and water state of Salix gordejivii. Journal of Desert Research (中国沙漠), 19 (Suppl. 1), 69-71. (in Chinese with English abstract) 